\title{
Raman investigation of local photo-bleaching in TDBC dye layer for photonics applications
}

\author{
Alban Gassenq, ${ }^{1, *}$ Yves Pipon, ${ }^{2}$ Gilles Montagnac, ${ }^{3}$ Olivier Boisron, ${ }^{1}$ \\ Valérie Martinez, ${ }^{1}$ Antoine Bard, ${ }^{1}$ Jean-Michel Benoit, ${ }^{1}$ Clémentine \\ Symonds, ${ }^{1}$ and Joel Bellessa ${ }^{1}$
}

\author{
Iniv Lyon 1, Institut Lumière Matière, UMR5306, CNRS, F-69622, LYON, France. \\ ${ }^{2}$ Univ Lyon 1, Institute of Physics of the 2 Infinities, UMR 5822, CNRS/IN2P3, F-69622, LYON, France. \\ ${ }^{3}$ Ecole Normale Supérieure de Lyon, Laboratoire de Géologie de Lyon, UMR CNRS 5276, CNRS, F-69364, LYON, France.
}

\begin{abstract}
Local photo-bleaching in dye layers is a promising method to pattern organic emitters for photonics applications like strong coupling researches or wavelength selective grating fabrication with TDBC J-aggregate layers. However, the understanding of the material change with bleaching in such layers is still ambiguous which limits this method to fully exploit its potential. Raman spectroscopy is a fast, non-destructive and readily technique to probe a material with micrometer spatial resolution but the conditions to explore bleached TDBC layers are not well known. In this work we have investigated active and bleached TDBC layers by Raman spectroscopy at different wavelengths in correlation with their optical properties. For active TDBC, Raman vibrations are well evidenced only in resonant configuration with suitable probe wavelengths (i.e when layer absorption is high and emission is low). For bleached TDBC, since layer absorption is limited to the UV range, Raman peaks are observed only under UV illumination with similar transitions compared to active layer which indicates that TDBC molecules are barely affected by the bleaching mechanism, even if the optical properties are changed around the fundamental transition. Such assumption was confirmed by XPS measurements indicating close stoichiometry and limited changes in the $\mathrm{N}$ chemical bonds between active and bleached material. This study allows a better understanding of the local photobleaching in TDBC dye layer for photonics applications and highlights the deep UV Raman spectroscopy as relevant tool for studying bleached organic emitters.
\end{abstract}

\section{Introduction}

Photo-bleaching is usually considered as a limitation for organic emitters $^{[1,2]}$. It consists in a photochemical alteration of a molecule which temporary or permanently becomes unable to fluoresce. Such modifications in covalent bonds are usually associated to transition from a singlet to triplet state and are fully dependent of the molecule and its environment ${ }^{[3]}$. Advantage can also be taken from photobleaching like for mobility measurement in biology [4] or for Tetrachloro Diethyl Benzimidazolo Carbocyanine (TDBC) dye. For TDBC J-aggregated layers, photo-bleaching is widely used for strong coupling studies ${ }^{[5-9]}$ and more recently for wavelength selective grating fabrication ${ }^{[10]}$. Indeed, TDBC dye layers have very interesting optical properties since they have a high oscillator strength at around $590 \mathrm{~nm}$ wavelength ${ }^{[11-13]}$ which can be suppressed under illumination ${ }^{[6]}$. Therefore, using local insolation, photo-bleaching can thus both spectrally and spatially change the refractive index with very high contrast at the micro-scale ${ }^{[10]}$. This property could be also exploited for other applications like light concentration ${ }^{[11]}$ or integrated optics since the refractive index contrast is even higher ${ }^{[14]}$ than usual materials ${ }^{[15]}$. However, this material modification is not yet well understood and thus cannot be fully exploited to develop new photonics devices.

On another hand, it has been shown that Raman spectroscopy is an efficient technic to probe TDBC molecule ${ }^{[16]}$ but only without photobleaching. Therefore, comparing active and bleached TDBC by Raman spectroscopy is of great interest for the understanding of the bleaching mechanism but such study is quite challenging due to the radical change in the optical properties of the layers before and after photo-bleaching. Therefore, Raman spectroscopy in correlation with the layer optical properties has to be studied.

In this paper, we present a detailed multi-wavelength Raman spectroscopy study on bleached and non-bleached TDBC (i.e active TDBC) with associated characterizations (absorption, photoluminescence and X-ray Photoelectron Spectroscopy). Such study allows thus a better understanding of the local photo-bleaching in TDBC dye layer which confirms and highlights the high potential of this method for photonics applications. Furthermore, it also shows a path of work for understanding the transitions from active to bleached molecule for other bleachable organic materials.

\section{Fabrication}

Samples consist of locally photo-bleached dye deposited on fused silica substrate (Suprasil $\mathrm{SiO}_{2}$.) Figure 1 presents the process flow for the sample fabrication. An aqueous solution of J-aggregated dye TDBC (5,6-Dichloro-2-[[5,6-dichloro-1-ethyl-3-(4-sulfobutyl)-benzimidazol2-ylidene]-propenyl]-1-ethyl-3-(4-sulfobutyl)-benzimidazolium hydroxide, inner salt, sodium salt) is spin coated on top of a $\mathrm{SiO}_{2}$ substrate at $2500 \mathrm{rppm}$ (Figure 1-a). The concentration of the TDBC aqueous solution is $8.39 \times 10^{-3} \mathrm{M}$. The spin coated layer has a $17 \mathrm{~nm}$ thickness evaluated by plasmon fitting ${ }^{[6,17]}$. For the insolation (Figure 1-b) laser writer ${ }^{[10]}$ or Nikon optical microscope was then used to 
locally insolate the layer. After insolation, regions which have been exposed to the light are locally photo-bleached (Figure 1-c).
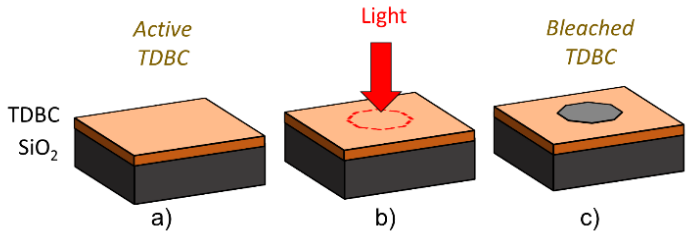

Fig. 1. Process flow for the sample fabrication starting from a glass substrate with first a) TDBC spin coating followed by b) the insolation giving c) locally-bleached layer.

Figure 2 presents top view image of TDBC layers observed with bright field imaging and x50 objective. Layers were insolated with $2 \mathrm{~mW}$ white light intensity focused over $2500 \mu \mathrm{m}^{2}$ in a microscope using the microscope diaphragm. Layers were compared without insolation (Figure 2-a), for $1 \mathrm{~min}$ insolation given $1 \mathrm{~kJ} / \mathrm{cm}^{2}$ dose (Figure 2-b) and for $10 \mathrm{~min}$ given $10 \mathrm{~kJ} / \mathrm{cm}^{2}$ dose (Figure 2-c). For low insolation dose (Figure 2-b), we clearly see that the layer is not fully photo-bleached since the image contrast between the insolated and un-insolated regions is relatively low. For high insolation dose (Figure 2-c), we assume a complete photo-bleaching since the image contrast is maximal.

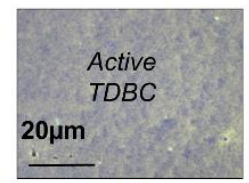

a)

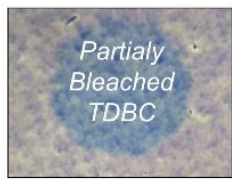

b)

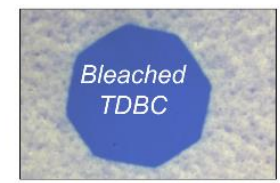

c)
Fig. 2. Top view imaging for a) active, b) partially bleached and c) fully bleached dye layer using a microscope setup for the insolation

\section{Optical properties}

In order to understand the different Raman signals, which will be presented later on in this work, we have first carefully studied the optical properties of the active and bleached TDBC layers by fluorescence and absorption measurements. For both active and bleached TDBC $17 \mathrm{~nm}$ TDBC layers were deposited on Suprasil substrate to avoid substrate UV absorption. Few $\mathrm{mm}^{2}$ surface were insolated at high dose and controlled by optical microscopy showing high contrast between both regions (Figure 2-c).

\section{TDBC optical properties}

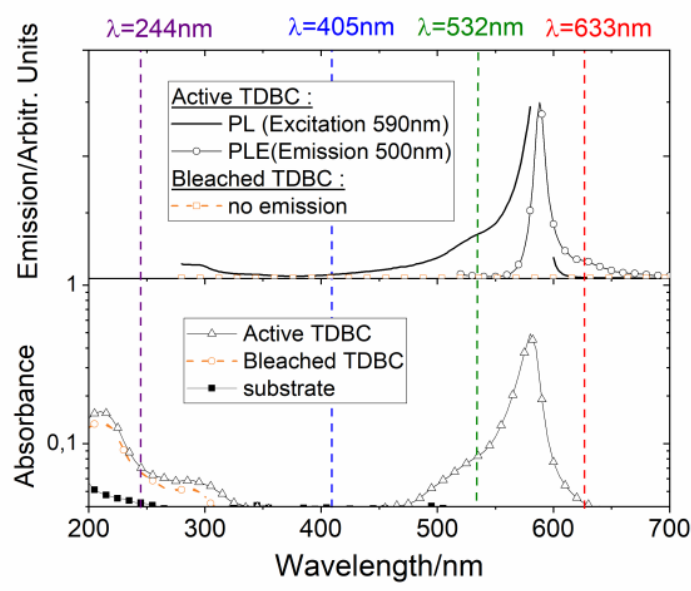

Fig 3. Active TDBC optical properties with a) spectrophotoluminescence and b) absorbance measurement

Figure 3 presents the emission (Figure 3-a) and absorption (Figure 3-b) properties of the TDBC layers for both bleached and active TDBC, the vertical dashed lines correspond to the Raman spectroscopy probe wavelengths which will be used later on in this work.

Emission properties (PL and PLE on the Figure 3-a) were measured with a visible FS5 Edinburgh spectro-fluorometer with a Xe lamp and photo-multiplication detector from 280 to $700 \mathrm{~nm}$. Photoluminescence spectrum was measured at $500 \mathrm{~nm}$ excitation wavelength and excitation spectrum was recorded at $590 \mathrm{~nm}$. For bleached TDBC, no significant emission was detected. For active TDBC, we clearly see that the maximum of the emission is obtained at around $590 \mathrm{~nm}$, as expected ${ }^{[10]}$, only under UV or between $\sim 500$ and $600 \mathrm{~nm}$ pumping. Therefore, we expect that fluorescence will potentially disturb the Raman spectra only for green pumping since no signal for the excitation spectra is found at 405 and $633 \mathrm{~nm}$ and that the fundamental emission (i.e 590nm) is far from the UV pump.

Absorbance measurements (Figure 3-b) were also measured with a Perkin-Elmer Lambda1050+ spectrometer for active and bleached TDBC. For active TDBC, two strong absorption windows are detected (in the UV and at around 590nm wavelength) which will allow to probe the active TDBC only with 244,532 or $590 \mathrm{~nm}$ wavelength. For bleached TDBC, only the UV absorption is detected. In that case, the UV probe will be suitable to measure the bleached TDBC by Raman spectroscopy since no absorption is detected for the other used wavelengths. To sum up, we have studied the optical properties of active and bleached TDBC. The deduced information from these measurements will be fundamental for the understanding of the Raman spectroscopy measurements presented below.

\section{Raman spectroscopy}

Raman spectroscopy measurements were performed at 4 different probe wavelengths in active and bleached layers. Furthermore, the Suprasil substrate was also measured without layer to understand the $\mathrm{SiO}_{2}$ contribution in the measured spectra. For visible probes (Figure 4-a, b and c), Raman spectroscopy analysis were performed on a Renishaw Invia Qontor with $1 \mathrm{~cm}^{-1}$ resolution using three lasers (405, 532 and $633 \mathrm{~nm}$ wavelength). Great care has been taken to ensure that the laser does not induce layer degradation during the measurements. Indeed, Raman spectra were recorded at low laser dose to avoid layers photo-bleaching (i.e power below $0.5 \mathrm{~mW}$ focused over few $\mu \mathrm{m}^{2}$ with $10 \mathrm{~s}$ acquisition time except for UV measurement were the spot moved at $50 \mu \mathrm{m} / \mathrm{s}$ ). Note that since the light dose given photo-bleaching is potentially dependent of the light intensity and spectra, the layers non-degradation was confirmed by direct microscopic fluorescence and bright field observation after the measurement and/or by performing several measurements on the same location given the same Raman spectra.

First of all, substrate Raman bands are detected for all wavelengths in good agreement with the literature with the expected main transitions at $\sim 800$ and $1100 \mathrm{~cm}^{-1[18]}$ (dashed vertical lines with \# symbols in the Figure 4). Regarding the TDBC layers, signals are completely different as a function of the wavelength probe. For blue probes (Figure 4-a), since no absorption is detected at such wavelength (Figure 3-b), no Raman signal is detected for both active and bleached TDBC. For green and red probes (Figure 4-b and c), the used wavelengths are close to the fundamental transition of TDBC at $590 \mathrm{~nm}$ (Figure 3), we are thus in resonant configuration for active TDBC giving enhanced Raman peaks. However, as indicated by the previous PLE 
measurements (Figure 3-a) green pump gives strong emission which prevents to clearly detect the TDBC transitions even if few small peaks (highlighted in the inset of the Figure 4-b) are nevertheless detected for the main TDBC transition at 662 and $1189 \mathrm{~cm}^{-1[16]}$. For $633 \mathrm{~nm}$ wavelength, strong Raman peaks are detected for active TDBC in good agreement with the literature (solid vertical lines with * symbols in the Figure 4). Note that each peak can be assigned to a vibration mode thanks to the DFT (Density Functional Theory) calculations from Ref. [16]. Indeed, such wavelength is perfectly suitable for TDBC Raman spectroscopy since it is close to the fundamental TDBC transition and does not give luminescence signal as indicated by the excitation curve presented in the Figure 3-a. However, for bleached TDBC, no Raman peaks are detected since no visible absorption exist at such wavelengths (i.e 533 and $633 \mathrm{~nm}$ ) as indicated by absorption measurements for bleached TDBC (Figure 3-b).

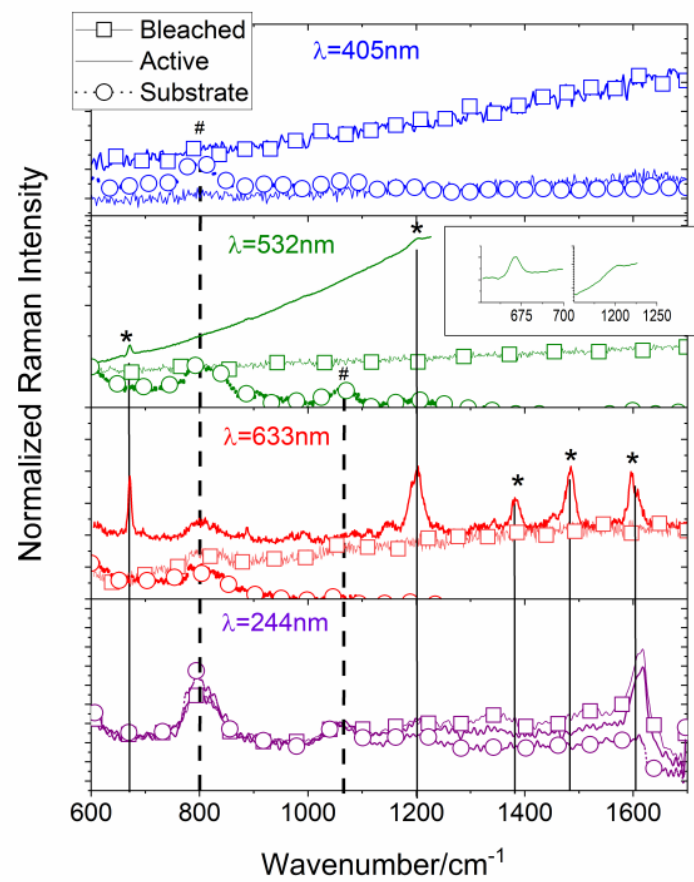

Fig. 4. Raman spectroscopy measurements performed with a) blue, b) green, c) red and d) UV laser probes

Deep UV Raman spectroscopy was then performed with $244 \mathrm{~nm}$ laser with $1 \mathrm{~cm}^{-1}$ resolution. In order to avoid sample bleaching or burning, the sample was moved during the measurement ${ }^{[19]}$ with $50 \mu \mathrm{m}$ step/s giving no layer degradation controlled by microscopy after the measurement. The Figure 4-d presents the back scattering signal with UV probes. In that case, only the transition at around $1600 \mathrm{~cm}^{-1}[16]$ is strongly observed since the phonon/electron couplings are highly dependent of the probe wavelength ${ }^{[2,21]}$, in resonant configuration ${ }^{[22,23]}$. Such transition is observed for both active and bleached TDBC.

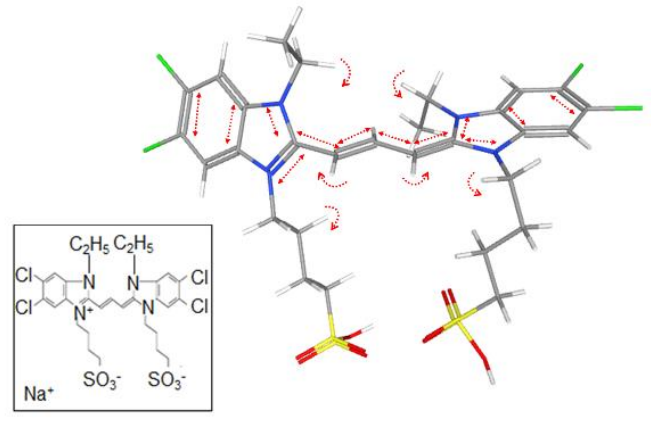

Fig. 5. Active TDBC molecule with the indicated Raman vibration mode detected for both bleached and active TDBC at $1600 \mathrm{~cm}^{-1}$ [16,24]

Figure 5 presents the corresponding detected Raman vibration at $\sim 1600 \mathrm{~cm}^{-1[16]}$ which is detected for both bleached and active TDBC in the UV range. Such mode is here presented on the active TDBC molecule ${ }^{[24]}$. It is directly related to benzene, imidazole rings and conjugate alkene chain vibration. Since this vibration is located in the center of the molecule and does not change between bleached and active TDBC molecule, we demonstrate here that the shape of the molecule is not completely changed or burned by the photo-bleaching mechanism which is in good agreement with the UV absorption which remains the same after bleaching. In order to confirm such assumption and to explore in more detail the photo-bleaching switching, we also have performed XPS measurement in active and bleached layers.

\section{XPS measurement}

XPS measurements were performed with a VG CLAM IV spectrometer using $\mathrm{Mg} \mathrm{k} \alpha$ radiation. All spectra were recorded with a pass energy of $50 \mathrm{eV}$ and a step size of $1 \mathrm{eV}$ for wide scans and $0.1 \mathrm{eV}$ for high resolution spectra. Collected XPS data were analyzed using CASAXPS software and were calibrated using the most intense $\mathrm{C}_{1 \mathrm{~s}}$ peak with a fixed value of $285 \mathrm{eV}$. The $\mathrm{O}_{1 \mathrm{~s}}$ and $\mathrm{C}_{1 \mathrm{~s}}$ low binding peaks arise from sample holder. Figure 6 presents the XPS spectra for bleached and active TDBC. Figure 6-a presents the full spectra with low energy resolution and Figure 6-b presents a zoom on the interesting peaks corresponding to the main atomic bond in the TDBC molecule. Note that no XPS measurement in TDBC layer have been already reported in the literature to our knowledge.

\section{XPS Measurements}

b)

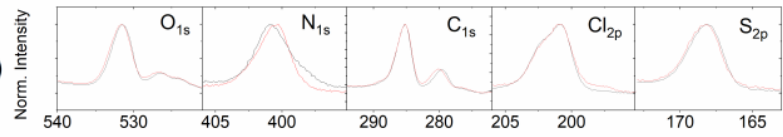

a)

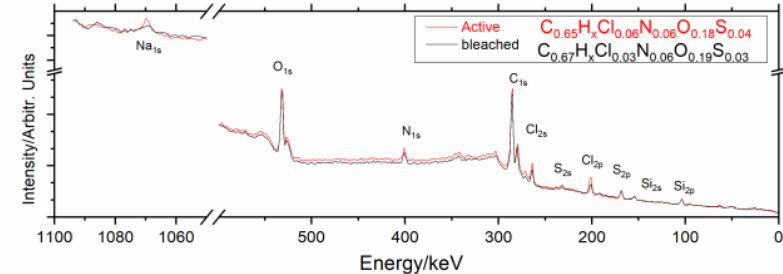

Fig. 6. XPS measurements performed in bleached and active TDBC layer for a) the full measured energy range and for b) different spectral regions at high resolution

From wide scans, stoichiometry measurements were performed after removing a Shirley background and using the appropriate sensitivity factors. The obtained results are indicated in the legend of the Figure 6b. We found a relatively good agreement with the theoretical stoichiometry of TDBC : $\mathrm{C}_{29} \mathrm{H}_{34} \mathrm{Cl}_{4} \mathrm{~N}_{4} \mathrm{O}_{6} \mathrm{~S}_{2}{ }^{[25]}$ given $\mathrm{C}_{0.64} \mathrm{H}_{\mathrm{x}} \mathrm{Cl}_{0.08} \mathrm{~N}_{0.08} \mathrm{O}_{0.13} \mathrm{~S}_{0.4}$ by taking into account that $\mathrm{H}$ composition cannot be accessed by XPS measurement. Furthermore, no strong change is detected between bleached and active stoichiometry since the setup reliability have been evaluated to $\pm 1 \%$ from time to time measurements. Regarding the binding energies of the peaks (Figure 6b) related to the atoms chemical environments ${ }^{[26]}$, except for $\mathrm{N}$ atoms, the peaks locations do not change confirming that no fundamental changes are detected in the molecule with bleaching even for sulfur atoms which was not measured by Raman spectroscopy since no detected Raman mode are associated to $S$ atoms ${ }^{[16]}$. For the small detected shift for the $\mathrm{N}_{1 \mathrm{~s}}$ spectrum maximum on the Figure 6-b $(\sim 0.5 \mathrm{eV})$, it can be attributed to the spectral shoulder change at lower energy with photo-bleaching. This could indicate that only few 
Nitrogen $(\mathrm{N})$ bonds are modified which could correspond to the only charged $\mathrm{N}$ atoms which is obviously less stable (see the molecule sketch in Figure 5). Anyway, XPS measurements confirm the Raman spectroscopy conclusion showing that the TDBC molecule is barely affected by the photo-bleaching. Furthermore, it also gives supplementary information showing that a limited change occurs for $\mathrm{N}$ bonds.

\section{Conclusion}

To sum up, we have performed multi-wavelength Raman spectroscopy in active and bleached TDBC layer in correlation with their optical properties. Absorption measurement exhibits two absorption regions: one at 220 and the other at $590 \mathrm{~nm}$ which is the only one which disappears with photo-bleaching. Furthermore, Photo-Luminescence (PL) and Photo-Luminescence Excitation (PLE) measurements exhibit emission at around $590 \mathrm{~nm}$ wavelength as expected ${ }^{[27]}$ which can be excited up to $\sim 610 \mathrm{~nm}$. Therefore, by studying these optical properties, we first shown that the probe wavelength is a key-feature for the measurement of active and bleached TDBC layer by Raman spectroscopy. We have then studied these layers by Raman spectroscopy at different wavelengths: 244, 405, 532 and $633 \mathrm{~nm}$. For active TDBC, strong Raman transitions are evidenced only with suitable probes (i.e when layer absorption is high and emission is low) at 633 and $244 \mathrm{~nm}$. For bleached TDBC, since layer absorption exists only in Ultra-Violet (UV) range as indicated by absorption measurements, the Raman transitions are observed only under UV illumination with a similar transition compared to active layer indicating limited changes in the shape of the molecule. This assumption was finally confirmed by X-ray Photoelectron Spectroscopy (XPS) measurements indicating similar stoichiometry and small change in the nature of an $\mathrm{N}$ atomic bonds after bleaching. Therefore, this work indicates that TDBC molecule is only slightly affected by the photo-bleaching mechanism even if optical properties are radically changed at around $590 \mathrm{~nm}$ wavelength ${ }^{[10]}$. These observations can now explain the fact that the refractive index is not changed by the photo-bleaching out of the fundamental energy at $\sim 590$ $\mathrm{nm}$ wavelength ${ }^{[6,10,14]}$. Such work allows thus a better understanding of the TDBC molecule photo-bleaching which is a promising technic for photonics applications. Moreover, this work highlights also the interest in performing multi-wavelength Raman studies including deep UV spectroscopy to understand the transition from active to bleached for organic emitters. Further investigations could be performed with complementary techniques (infrared spectroscopy, solid-state nuclear magnetic resonance, X-ray absorption near edge structure...) which remain challenging in such thin layers, with associated energy and molecular orbitals calculations.

Acknowledgment. The authors would like to thank the PLYRA, CECOMO and Nanolyon Platform for measurement and clean room facilities, Gamze Yavuz and Clotilde Gaillard for Raman measurements, and the ANR PlasHybrid project (ANR-18-CE300014). The authors are also grateful to the Raman facility in Lyon (France) supported by the Institut National des Sciences de l'Univers (INSU) and to the LABEX Lyon Institute of Origins (ANR-10LABX-0066) of the Université de Lyon for its financial support within the program "Investissements d'Avenir" (ANR-11-IDEX-0007) of the French government operated by the National Research Agency (ANR).

Disclosures. The authors declare no conflicts of interest.

\section{References}

[1] T. Ha, P. Tinnefeld, Annu. Rev. Phys. Chem 2012 , 63, 595.

[2] B. H. Cumpston, K. F. Jensen, Synth. Met. 1995, 73, 195.
[4] D. Axelrod, D. E. Koppel, J. Schlessinger, E. Elson, W. W. Webb, Biophys. J. , DOI:10.1016/S00063495(76)85755-4.

[5] B. Munkhbat, M. Wersäll, D. G. Baranov, T. J. Antosiewicz, T. Shegai, Sci. Adv. 2018, 4, eaas 9552 6.

[6] K. Chevrier, J. M. Benoit, C. Symonds, S. K. Saiki, J. Y. Zhou, J. Bellessa, Phys. Rev. Lett. 2019, 122, 173902.

[7] F. Stete, W. Koopman, M. Bargheer, ACS Photonics 2017, 4, 1669.

[8] N. Li, Z. Han, Y. Huang, K. Liang, X. Wang, F. Wu, X. Qi, Y. Shang, L. Yu, B. Ding, B. Ding, B. Ding, J. Mater. Chem. C 2020, 8, 7672.

[9] S. Nosrati, T. Rammler, A. J. Meixner, F. Wackenhut, J. Phys. Chem. C 2021, 125, 13024.

[10] A. Gassenq, K. Chevrier, A. Bard, J. Benoit, C. Symonds, J. Bellessa, A. Gassenq, K. Chevrier, A. Bard, J. Benoit, C. Symonds, J. M. Benoit, A. Gassenq, K. Chevrier, A. Bard, J. Benoit, C. Symonds, J. Bellessa, Appl. Opt.

[11] A. Cacciola, C. Triolo, O. Di Stefano, A. Genco, M. Mazzeo, R. Saija, S. Patanè, S. Savasta, ACS Photonics, DOI:10.1021/acsphotonics.5b00197.

[12] B. W. HOON, I. C. SEO, E. LEE, A. SOO-CHAN H. Y. JEONG, Opt. Express 2017, 25, 206.

[13] K. Takatori, T. Okamoto, K. Ishibashi, R. Micheletto, Opt. Lett. 2017, 42, 3876.

[14] A. Gassenq, K. Chevrier, A. Bard, J. Benoit, C. Symonds, in Accepted for publication in CLEO 2021 proceeding, 2021, vol. 59, p. 2482524.

[15] C. R. Doerr, Front. Phys. 2015, 3, 1.

[16] J. R. Blake, P. M. Colombera, Chem. Eng. Sci. 1977, 32, 221.

[17] C. Bonnand, J. Bellessa, J. C. Plenet, Phys. Rev. B Condens. Matter Mater. Phys. DOI:10.1103/PhysRevB.73.245330.

[18] M. Saito, F. Damico, F. Bencivenga, R. Cucini, A. Gessini, E. Principi, C. Masciovecchio, J. Chem. Phys., DOI:10.1063/1.4884155.

[19] G. Montagnac, J. Hao, U. Pedreira-Segade, I. Daniel, Appl. Clay Sci. 2021, 200, 105824.

[20] S. A. Asher, C. R. Johnson, Science (80-. ). 1984, 225,311 .

[21] G. R. Loppnow, L. Shoute, K. J. Schmidt, A. Savage, R. H. Hall, J. T. Bulmer, Philos. Trans. R. Soc. A Math. Phys. Eng. Sci. 2004, 15, 2461.

[22] A. C. Albrecht, J. Chem. Phys. 1961, 34, 1476.

[23] E. Quirico, G. Montagnac, V. Lees, P. F. McMillan, C. Szopa, G. Cernogora, J. N. Rouzaud, P. Simon, J. M. Bernard, P. Coll, N. Fray, R. D. Minard, F. Raulin, B. Reynard, B. Schmitt, Geochim. Cosmochim. Acta 2020, 282, 156.

[24] TDBC - PubChem, National Library of Medecine.

[25] S. K. Saikin, A. Eisfeld, S. Valleau, A. AspuruGuzik, Nanophotonics, 2013.

[26] P. van der Heide, X-Ray Photoelectron Spectroscopy: An Introduction to Principles and Practices, 2011.

[27] C. Symonds, C. Bonnand, J. C. Plenet, A. Bréhier, R. Parashkov, J. S. Lauret, E. Deleporte, J. Bellessa, New J. Phys. 2008, 10, 065017. 